Rev. Elev. Méd. vét Pays trop., 1971, 24 (2) : 173-81

\title{
Résultats d'une étude sur la valeur comparée du lauryl sulfate de sodium et du bromure de cetylpyridinium pour l'isolement de Mycobactéries à partir de prélèvements animaux et humains
}

\author{
par J. CHAMBRON (*) et H. SARRAT (**)
}

\begin{abstract}
RESUME
Les auteurs donnent les résultats dune enquête comparative du lauryl sulfate de sodium alcalin et du bromure de cétylpyridinium comme agents d'homogénéisation et de décontamination pour l'isolement de mycobactéries sur les milieux à l'œuf.

Ces deux produits sont expérimentés sur 25 prélèvements d'origine humaine (crachats) et sur 26 prélèvements d'origine animale (ganglions à tubercules caséeux ou infectés artificiellement).

La technique utilisant le lauryl sulfate alcalin se montre la plus favorable, pour les deux types de prélèvements. Ses avantages compensent largement les légers inconvénients qu'entraîne la nécessité d'une neutralisation.

Grâce à sa faible toxicıté, le cétylpyridinium conserve tout son intérêt en Afrique comme agent de transport des prélèvements récoltés loin du laboratoire.
\end{abstract}

\section{INTRODUCTION}

L'efficacité d'une méthode d'isolement des mycobactéries sur milieux à l'œuf classiques dépend essentiellement des qualités de lagent de décontamination utilisé. De nombreux produits sont tout d'abord essayés dans différents pays selon des modalités très variées : hydroxyde de soude (Petroff), carbonate d'ammonium (Goldie), acide chlorhydrique (Mac Nab), phosphate tri-sodique (Corper et Stoner), acide oxalique (Corper et $\mathrm{U}$. yei), acide sulfurique (Saenz et Costil), etc. Aucun d'eux ne donne entièrement satisfaction car tous se montrent encore trop toxiques vis-à-vis du bacille de Koch. Seule se dégage progressivement une méthode efficace de traitement des prélève-

(*) Laboratoire national de l'Elevage et de Recherches vétérinaires de Dakar-Hann.

(**) Institut Pasteur de Dakar. ments pathologiques, faisant appel à une homogénéisation acide ou alcaline, suivie d'une neutralisation puis d'une centrifugation.

En France, l'hydroxyde de soude et l'acide sulfurique gardent pendant longtemps la faveur des bactériologistes. Mais l'apparition de corps chimiques nouveaux révélant des propriétés très intéressantes relance l'intérêt des chercheurs. GERNEZ-RIEUX, BETHOUART et TACQUET étudient l'action des ammoniums quaternaires (3). TACQUET, TISON, avec divers collaborateurs, expérimentent certains détergents tensio-actifs, le Teepol 410 basique $(11,6)$ puis, lorsque sa fabrication est arrêtée, le lauryl sulfate de sodium (7). Des études comparatives de ce dernier avec les décontaminants classiques ou des produits étrangers (Sipon, Rodalon, Nekal BS, ...) et la mise au point d'une technique efficace font ainsi l'objet de diverses publications $(8,9,13)$. En 1968, 
LANGEROVA et TACQUET (4) le testent vis-à-vis de 8 autres produits. Tous ces travaux démontrent la supériorité du lauryl sulfate de sodium alcalin pour l'isolement des mycobactéries sur milieu à l'œuf à partir des crachats. Son usage est étendu à la recherche de ces mêmes germes dans le lait, les produits laitiers (10) et dans les viandes (12).

Mais il est un autre produit, expérimenté en Allemagne fédérale en 1960, le bromure de cétylpyridinium (1) qui, très utilisé à l'étranger, est largement employé depuis de nombreuses années en Afrique francophone par les principaux laboratoires étudiant les mycobactéries. Cet ammonium quaternaire possède des propriétés très intéressantes pour les pays chauds, en particulier celle d'être un bon milieu de transport pour les prélèvements destinés au laboratoire. Il se montre, en effet, très peu nocif vis-à-vis du bacille tuberculeux, même au cours d'un contrat prolongé à la température ambiante, généralement élevée en Afrique.

En dépit de ses qualités dignes d'attention, aucune étude qualitative comparée entre le lauryl sulfate et le bromure de cétylpyridinium n'a jusqu'ici été, à notre connaissance, publiée.

C'est pour combler cette lacune que nous nous sommes attachés à comparer ces deux produits, en opérant, dans des conditions rigoureusement identiques, sur les mêmes prélèvements animaux (broyat d'organes) et humains (crachats).

\section{MATERIEL - METHODES}

\section{Matériel}

L'expérimentation porte sur 51 prélèvements, soit 26 d'origine animale et 25 d'origine humaine.

\subsection{Prélèvements d'origine animale}

Ils sont tous récoltés à l'abattoir de Dakar sur des animaux apparemment sains et comprennent cinq ganglions préscapulaires et trachéo-bronchiques de bovins et 21 ganglions mésentériques de porcs. Cinq prélèvements d'origine porcine présentent des tubercules caséeux multiples et sont positifs à l'examen optique après coloration (présence de bacilles acido-alcoolo-résistants). Les autres ganglions ne présentent aucune lésion anatomo-pathologique visible.

\subsection{Prélèvements d'origine humaine}

Ils sont constitués par 25 crachats humains fortement positifs à l'examen optique (présence de très nombreux bacilles acido-alcoolo-résistants).

\section{Méthodes}

\subsection{Préparation des prélèvements d'origine animale}

Chaque fois que cela est possible, les cultures comparatives sont effectuées à partir de ganglions provenant d'animaux naturellement infectés ( 5 prélèvements de porcs). Les cas de tuberculose étant rares à l'abattoir de Dakar, les autres analyses portent sur des broyats ganglionnaires contaminés artificiellement au Laboratoire. La technique est la suivante : broyage du ganglion au mortier en présence d'eau distillée et de sable stérile -- récolte du liquide surnageant - les ganglions sans lésions apparentes sont contaminés artificiellement par adjonction de deux öses de raclage d'une culture jeune (10 à 20 jours d'étuve) de $M y c o b a c$ terium tuberculosis suivie d'une homogénéisation soignée dans un microbroyeur M.S.E. Deux parties égales de $2 \mathrm{ml}$ chacune, exactement mesurées à la pipette de précision, sont prélevées sur le volume total de liquide surnageant. L'une sera traitée par le lauryl sulfate alcalin, l'autre par le bromure de cétylpyridinium.

\subsection{Préparation des prélèvements d'origine humaine}

On prélève de la même façon deux parties égales de $2 \mathrm{ml}$ chacune, qui sont traitées comme ci-dessus.

\subsection{Traitement par le lauryl sulfate de sodium sodique (L.S.S.)}

Il est pratiqué selon la méthode préconisée par TACQUET et TISON $(7,9)$ :

a) Homogénéisation - décontamination

Deux ml de liquide surnageant ou de crachat sont additionnés de $3 \mathrm{ml}$ (exactement mesurés à la pipette) de la solution suivante:

- lauryl sulfate de sodium pur ( $\left.{ }^{1}\right)$ pour analyse

- hydroxyde de sodium pur en pastilles

$10 \mathrm{~g}$

(1) SERLABO - 26, rue Saint-Gilles, 75 - Paris 3e. 
- eau distillée - q.s.p.

$1.000 \mathrm{ml}$

(dissoudre le lauryl dans l'eau chaude et ajouter ensuite l'hydroxyde de sodium. Conserver la solution à l'étuve à $37^{*}$ ).

Pour éviter les souillures de parois, le mélange est transvasé dans un second tube à centrifuger conique stérile, d'au moins $60 \mathrm{ml}$ et bouchant hermétiquement; il est agité $30 \mathrm{minu}-$ tes avec un agitateur mécanique (appareil utilisé : agitateur oscillant Prolabo).

b) Lavage - neutralisation

La quantité nécessaire de la solution suivante (stérilisée à l'autoclave) :

- acide phosphorique

- pourpre de bromocrésol à $1 / 250$

$1,5 \mathrm{ml}$

— eau distillée - q.s.p.

$2 \mathrm{ml}$

est versée progressiv est versée progressivement sur le produit homogénéisé alcalin, de couleur bleue, jusqu'au virage au jaune paille (retour à une légère acidité, avec formation d'un précipité discret facilitant la centrifugation des germes).

c) Centrifugation 30 minutes à $3.000 \mathrm{t} / \mathrm{mn}$

Le surnageant est rejeté et le culot parfaitement égoutté.

Toutes ces opérations sont faites aseptiquement.

\subsection{Traitement par le bromure de cétylpyridinium ${ }^{(2}$ )}

Il est pratiqué selon la méthode de BERENCSI, HIDEG et VECSEY (1) :

a) Homogénéisation - décontamination

A 1 volume de crachat ou de liquide surnageant, on ajoute 5 volumes de B.C.P. en solution aqueuse à 1 p. 100 . Le mélange, transvasé en tube à centrifuger à fermeture hermétique, est agité 15 minutes, puis porté dans une étuve à $37^{\circ}$ pendant 24 heures.

b) Centrifugation: au bout de ce temps, on procède de la même façon que pour le L.S.S.

\subsection{Ensemencement - cultures - expression des résultats}

On ajoute la quantité d'eau distillée juste suffisante pour ensemencer la totalité du culot sur le nombre de tubes choisis et pour pratiquer les dilutions nécessaires. A la suite de

() Bromure de cétylpyridinium no $2386 / \mathrm{B}$ - B.B.L. - 33, rue Voltaire, Puteaux - Seine. quelques tâtonnements, nous avons adopté la méthode quantitative suivante:

- Pour chacun des prélèvements de crachats, richement contaminés, on pratique des dilutions décimales jusqu'à $10^{-5}$. Seules les dilutions $10^{-1}, 10^{-3}, 10^{-5}$ sont ensemencées sur milieu de base de Coletsos, à raison de 4 tubes par dilution, et de $0,2 \mathrm{ml}$ exactement mesurés à la pipette graduée dans chacun des tubes.

- Pour les prélèvements d'origine animale, on effectue des dilutions décimales jusqu'à $10^{-3}$. Le surnageant non dilué et chacune des dilutions sont ensemencés, chacun sur cinq milieux de Lowenstein-Jensen $(0,2 \mathrm{ml}$ par tube).

Les tubes sont gardés au moins 15 jours en position horizontale (bouchon légèrement dévissé pendant 24 heures) puis redressés. Les cultures sont conservées deux mois à l'étuve à $37^{\circ}$.

La richesse des cultures est évaluée arbitrairement de la façon suivante :

- 0 à 200 colonies : comptage exact des colonies;

- 200 à 400 colonies : comptage approximatif des colonies;

- incomptable (culture en nappe) : $\geqslant 500$ (500 colonies ou plus).

Pour chaque dilution, le résultat est exprimé en nombre moyen de colonies par tube, calculé sur l'ensemble des tubes de cette dilution. En cas de cultures en nappes, ce nombre moyen est précédé du signe $\geqslant$ (nombre de colonies égal ou supérieur à ...).

\section{RESULTATS}

(Abréviations utilisées: L.S.S. = lauryl sulfate de sodium alcalin - B.C.P. = bromure de cétylpyridinium).

Selon l'obtention de cultures à des dilutions semblables ou différentes d'une part, et selon la richesse de ces cultures aux mêmes dilutions d'autre part, les résultats peuvent être classés en trois catégories:

- un des deux produits permet d'obtenir des cultures à des dilutions plus élevées, et se montre ainsi très nettement supérieur à l'autre; 
- un des deux produits permet d'obtenir des cultures plus riches, aux mêmes dilutions, et se montre plus favorable que l'autre;

- les deux produits donnent des résultats sensiblements identiques.

Les chiffres indiqués dans les tableaux suivants représentent le total des nombres moyens de colonies et le nombre de prélèvements à colonies en nappe incomptable. Ce dernier chiffre complète le premier, surtout pour les dilutions les plus faibles à culture en nappe où l'erreur par défaut est souvent importante, le nombre réel de colonies dépassant largement le nombre de 500 retenu arbitrairement.

\section{Prélèvements d'origine animale}

Au total, 26 prélèvements : 3 donnent des résultats inutilisables (1 est souillé et 2 autres dont 1 prélèvement pathologique ne montrent aucune culture), 23 donnent des résultats exploitables (4 prélèvements pathologiques et 19 contaminés artificiellement).

1.1. L.S.S. très nettement supérieur au B.C.P. : 13 prélèvements sur 23 (dont 3 prélèvements pathologiques sur 4).

TABLEAU N $\mathrm{N}^{\circ} \mathrm{I}$

\begin{tabular}{|c|c|c|c|c|c|c|c|c|}
\hline & \multicolumn{2}{|l|}{ Pur } & \multicolumn{2}{|c|}{$10^{-1}$} & \multicolumn{2}{|c|}{$10^{-2}$} & \multicolumn{2}{|c|}{$10^{-3}$} \\
\hline $\begin{array}{l}\text { L.S.S. } \\
\text { Total des colonies } \\
\text { incomptable }\end{array}$ & $\geqslant 2912$ & 5 & $\geqslant 1249,2$ & 1 & $\geqslant 583,2$ & 1 & $\geqslant 506,6$ & 1 \\
\hline $\begin{array}{l}\text { B.C.P. } \\
\text { Total des colonies } \\
\text { incomptable }\end{array}$ & $\geqslant 1077,2$ & 1 & $\geqslant 158,8$ & 0 & $\geqslant 17,8$ & 0 & 0 & 0 \\
\hline
\end{tabular}

4 prélèvements (dont 2 pathologiques) sont négatifs avec le B.C.P. et positifs avec le L.S.S.
1.2. L.S.S. plus favorable que le B.C.P. : 7 prélèvements sur 23 (dont 1 prélèvement pathologique).

TABLEAU $N^{\circ}$ II

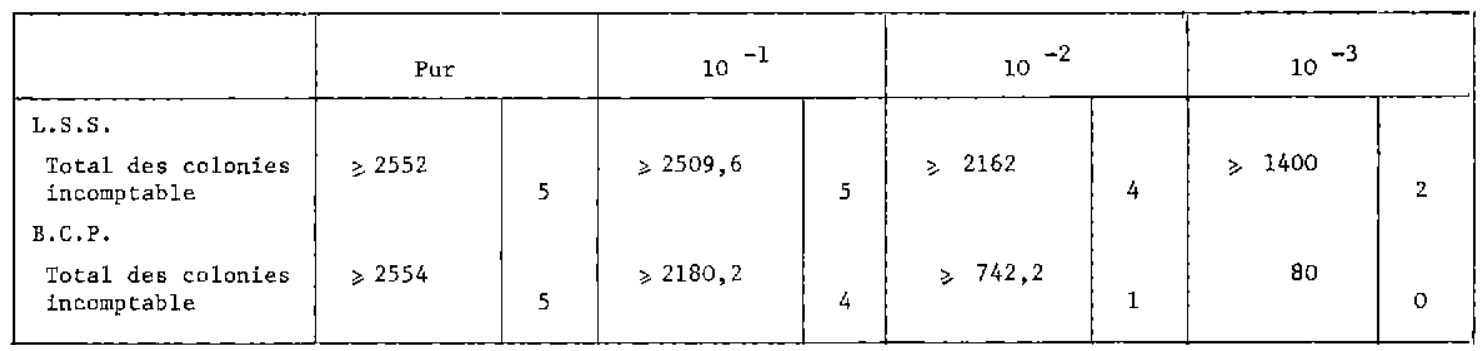

1.3. Résultats identiques avec le L.S.S. et le

B.C.P. : 2 prélèvements sur 23 .

TABLEAU $N^{\circ}$ III

\begin{tabular}{|c|c|c|c|c|}
\hline & Pur & $10^{-1}$ & $10^{-2}$ & $10^{-3}$ \\
\hline $\begin{array}{c}\text { L.S.S. } \\
\text { Total des colonies } \\
\text { B.C.P. } \\
\text { Total des colonies }\end{array}$ & 246,6 & 31 & 3 & 0 \\
\hline
\end{tabular}


1.4. B.C.P. plus favorable que le L.S.S. : 1 prélèvement sur 23

TABLEAU $\mathrm{N}^{\circ} \mathrm{IV}$

\begin{tabular}{|l|c|c|c|c|}
\hline & Pur & $10^{-1}$ & $10^{-2}$ & $10^{-3}$ \\
\hline $\begin{array}{l}\text { L.S.S. } \\
\text { Total des colonies }\end{array}$ & $\geqslant 500$ & $\geqslant 500$ & $\geqslant 174$ & $\geqslant 29$ \\
$\begin{array}{c}\text { B.C.P. } \\
\text { Tots1 des colonies }\end{array}$ & $\geqslant 500$ & $\geqslant 500$ & $\geqslant 500$ & 178 \\
\hline
\end{tabular}

\section{Prélèvements d'origine humaine}

$\mathrm{Au}$ total, 25 prélèvements : 4 donnent des résultats inutilisables ( 2 sont souillés, 2 autres ne montrent aucune culture), 21 sont exploitables.

2.1. L.S.S. très nettement supérieur au B.C.P. : 12 prélèvements sur 21.

TABLEAU $\mathrm{N}^{*} \mathrm{~V}$

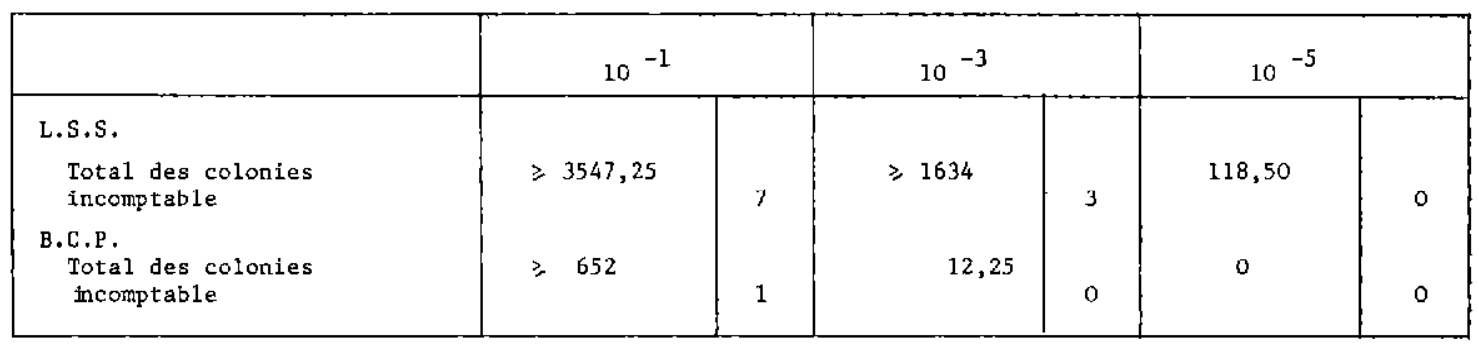

4 prélèvements sont négatifs avec le B.C.P. avec le B.C.P., et culture jusqu'à $10^{-5}$ avec le et positifs avec le L.S.S. (pour un cinquième L.S.S.). prélèvement, une seule colonie moyenne à $10^{-1}$

2.2. L.S.S. plus favorable que le B.C.P. : 6 prélèvements sur 21 .

TABLEAU $N^{\circ} \mathrm{VI}$

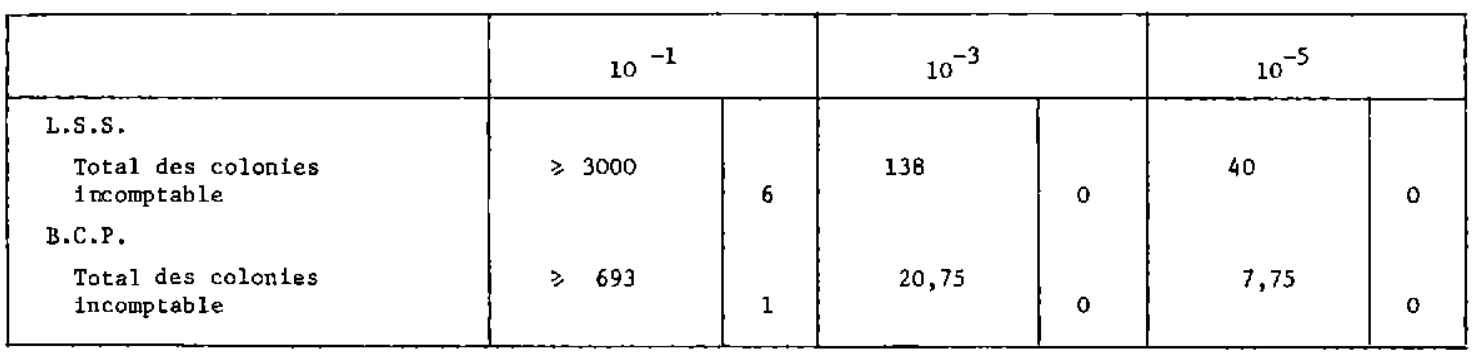


2.3. Résultats identiques avec le L.S.S. et le B.C.P. : 2 prélèvements sur 21 .

TABLEAU $\mathrm{N}^{\circ} \mathrm{VII}$

\begin{tabular}{|l|c|c|c|}
\hline & $10^{-1}$ & $10^{-3}$ & $10^{-5}$ \\
\hline $\begin{array}{l}\text { L.S.S. } \\
\text { Tota1 des colonies } \\
\text { B.C.P. } \\
\text { Total des colonies }\end{array}$ & 35 & 0 & 0 \\
\hline
\end{tabular}

3. Résultats généraux (addition des divers résultats)
2.4. B.C.P. très nettement supérieur au L.S.S. :

1 prélèvement sur 21 .

\section{TABLEAU $N^{*} I X$}

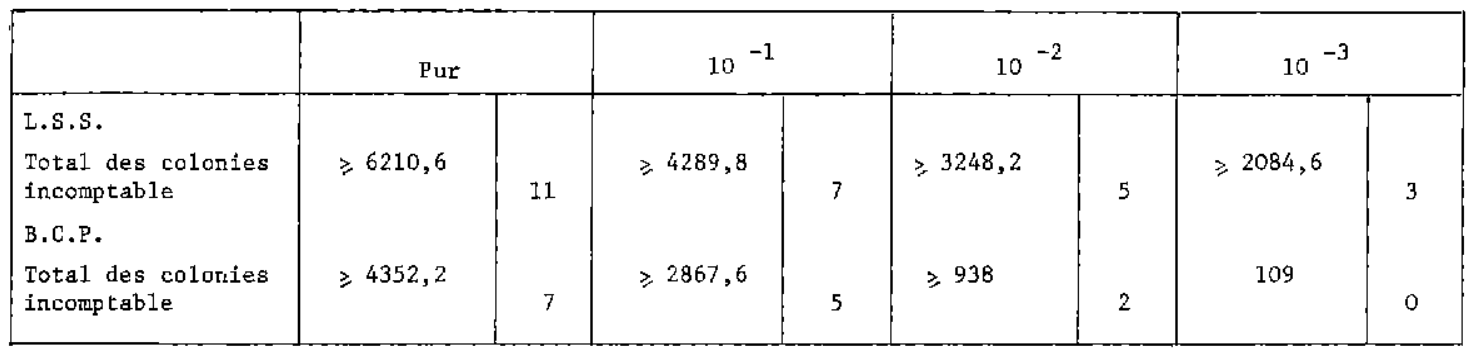

\subsection{Prélèvements d'origine humaine}

TABLEAU $\mathrm{N}^{\bullet} \mathrm{X}$

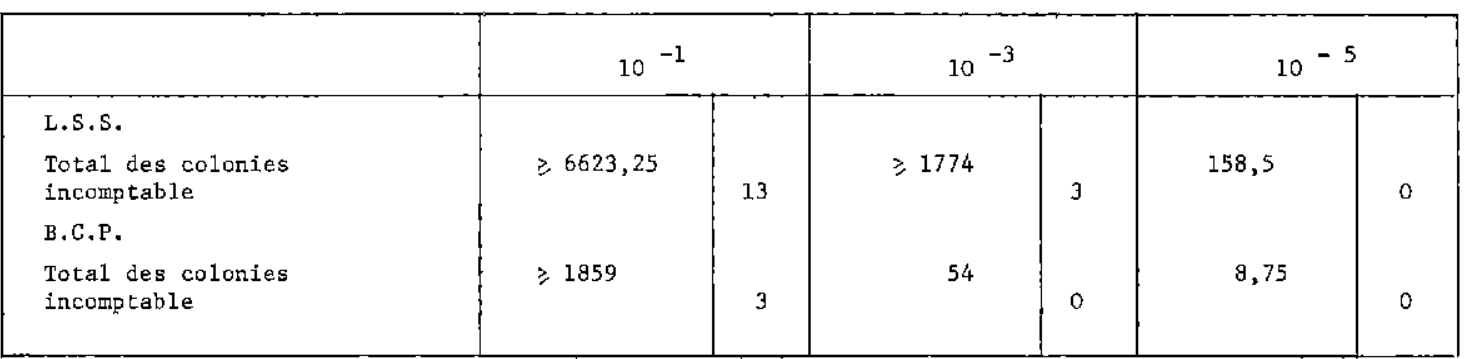

De l'examen de ces divers résultats, on peut conclure que le lauryl sulfate de sodium alcalin, utilisé selon la technique décrite, donne de meilleurs résultats que le bromure de cétylpyridinium pour isoler des mycobactéries par culture sur des milieux à l'œuf, à partir de produits pathologiques d'origine animale ou humaine. Ainsi, il permet la culture de $M$. tuberculosis
TABLEAU $\mathrm{N}^{\circ} \mathrm{VIII}$

\begin{tabular}{l|c|c|c|} 
& $10^{-1}$ & $10^{-3}$ & $10^{-5}$ \\
\hline $\begin{array}{l}\text { L.S.S. } \\
\text { Total des colonies } \\
\text { B.C.P. } \\
\text { Total des colonieg }\end{array}$ & 41 & 2 & 0 \\
$\begin{array}{l}\geqslant 500 \\
(1 \text { ncompt.) }\end{array}$ & 21 & 1 \\
\hline
\end{tabular}

3.1. Prélèvements d'origine animale 


\section{DISCUSSION}

\section{Interprétation des résultats chiffrés}

La méthode quantitative utilisée dans cette étude, comme toutes les méthodes de titrage actuelles, est basée sur la numération des colonies apparaissant sur les milieux à l'œuf après ensemencement de dilutions décimales jusqu'à $10^{-3}$ ou $10^{-5}$. Or, contrairement à ce qui se passe avec la plupart des autres germes microbiens, on sait combien cette numération est difficile et infidèle avec les mycobactéries, germes à dispersion malaisée. Les difficultés augmentent avec le nombre de colonies. C'est ce qui nous a incité, à l'exemple d'autres auteurs traitant du même sujet, à utiliser un système de notation arbitraire pour exprimer les résultats des cultures.

D'autre part, l'usage montre que les divers chiffres obtenus pour une même série de dilutions décimales sont souvent loin d'être proportionnels et conformes à ce qu'ils devraient être en fonction de ces dilutions. Ils sont souvent bien plus élevés qu'on ne l'attendait. Cela tient à la présence de gros amas de germes au sein du produit pathologique. Leur fragmentation, très incomplète avec les méthodes de broyage usuelles, est facilitée par les manipulations successives à l'occasion des dilutions et des ensemencements. Plusieurs auteurs font allusion à ce problème (2).

Nos prélèvements, principalement ceux d'origine humaine, n'échappent pas à ce phénomène. Les prélèvements contaminés artificiellement, du fait même de leur homogénéisation soignée dans un micro-broyeur, y semblent moins sensibles.

Compte tenu de ces remarques, les chiffres indiqués dans les tableaux de résultats sont très imprécis et ne peuvent donner lieu à aucun calcul statistique comparatif. Mais les différences constatées, bien qu'impossibles à évaluer avec rigueur, sont suffisantes pour reconnaître sans ambiguïté qu'un des deux produits donne des résultats nettement plus favorables que l'autre.

\section{Valeur comparative des deux produits Avantages et inconvénients de chacune des deux méthodes utilisées}

Les avantages du lauryl sulfate par rapport à divers autres produits (auxquels il convient d'ajouter maintenant le cétylpyridinium) ont déjà été précisés par TISON, TACQUET et leurs collaborateurs dans leurs publications. Nous ne ferons que les rappeler brièvement:

- meilleure homogénéisation, d'où examen microscopique amélioré;

- culture plus riche en colonies;

- décontamination meilleure;

- respect de la qualité du milieu à l'œuf (absence de protéolyse).

Un des inconvénients de la méthode au lauryl sulfate alcalin est qu'elle exige une neutralisation. Celle-ci améliore considérablement les résultats (8), mais représente une manipulation supplémentaire qui entraîne une perte de temps (surtout pour les cultures d'échantillons en série) et augmente les possibilités de contamination secondaire. Ces inconvénients nous semblent cependant mineurs: la neutralisation, basée sur le virage d'un indicateur coloré incorporé à la solution acide, est effectuée très rapidement. Le respect des conditions d'aseptie habituelles montre que les contaminations sont très rares. Nous pensons que l'augmentation sensible des résultats positifs compense largement les désavantages constatés.

Le bromure de cétylpyridinium se révèle un moins bon agent d'homogénéisation de décontamination que le lauryl sulfate.

Le grand avantage de la technique utilisant ce produit est sa plus grande simplicité, due à l'absence de neutralisation. Un autre avantage, plus réel à nos yeux, est que le cétylpyridinium se montre remarquablement peu nocif vis-à-vis du bacille de Koch. Diverses études sont en cours, conduites en particulier par l'un de nous à Dakar (5); elles montrent qu'on peut laisser agir ce produit sur des crachats à la température ambiante pendant plusieurs jours sans diminuer sensiblement le taux de positivité des cultures. On peut donc envisager son utilisation comme milieu de transport en Afrique et dans les pays chauds, dans les conditions du travail en brousse. Pour le travail de routine au laboratoire, cet avantage se révèle évidemment sans intérêt. 


\section{v. CONCLUSION}

Pour les isolements de mycobactéries par culture sur milieux à l'œuf, le lauryl sulfate donne de meilleurs résultats que le cétylpyridinium, quelle que soit l'origine humaine ou animale des prélèvements. Difficile à chiffrer, la comparaison est cependant nettement en faveur du premier produit.

La méthode préconisée par TACQUET, TISON et leurs collaborateurs et utilisant le lauryl sulfate de sodium en solution alcaline offre de légers inconvénients par rapport à la technique plus simple utilisant le bromure de cétylpyridinium. Ces désavantages sont largement compensés par une plus grande activité du premier produit, qui permet des cultures plus souvent positives, et plus riches.

Compte tenu de certaines de ses qualités, que nous avons rappelées, le bromure de cétylpyridinium offre malgré tout certains avantages qui le feront préférer parfois, notamment en Afrique, lorsque l'acheminement des prélèvements au laboratoire doit s'effectuer dans des conditions difficiles.

\section{SUMMARY}

\section{Results of a comparative study with alkaline sodium lauryl-sulfate and cetylpyridinium bromure for isolation of mycobacteria from animal and human products}

The autors give the results of a comparative study of these two chemical products for the homogeneization and decontamination of 25 spitles and 26 animals samples.

The alkaline sodium lauryl-sulfate technique, that needs a neutralization seems to be the most efficient for the isolation of mycobacteria by culture on egg media from both contamined human and animal products.

Advantages and disadvantages of each technique are reminded.

The cetylpyridinium bromure proved less efficient, but remains a good transport agent in Africa where laboratories are rare.

\section{RESUMEN}

Resultados de un estudio sobre el valor comparado del lauryl sulfato de sodio y del Bromuro de Cetylpyridinium para el aislamiento de micobacterias a partir de muestras animales y humanas

Los autores dan los resultados de una encuesta comparativa del lauryl sulfato de sodio alcalino y del bromuro de cetylpyridinium como agentes de homogeneización y de descontaminación para el aislamiento de micobacterias sobre los medios con huevo.

Se experimentaron dichos dos productos sobre 25 muestras de origen humana (esputos) y sobre 26 muestras de origen animal (ganglios con tuberculos caseosos o infectados artificialmente).

La técnica utilizando el lauryl sulfato alcalino es la más favorable para los dos tipos de muestras. Sus ventajas ampliamente compensan los pequeños inconvenientes que acarrea la necesidad de una neutralización.

A causa de su toxicidad poco importante, el cetylpyridinium conserva todo su interés en Africa como agente de transporte de las muestras recogidas lejos del laboratorio.

\section{BIBLIOGRAPHIE}

1. BERENCSI (G.), HIDEG (K.) et VECSEY (Z.), «L'emploi du cétylpyridinium pour l'homogénéisation dans le diagnostic bactériologique de la tuberculose », Zentralblatt Bakt, I Abt. Orig., 1960, 178 (3) : 332-36.

2. BRETTEY (J.), «Une nouvelle méthode de mesure de la résistance des mycobactéries ", Bull. Acad. nat. Méd., 1970, 154 (5-6) : 95-100.

3. GERNEZ-RIEUX (CH.), BETHOUART (J.) et
TACQUET (A.), «Technique d'isolement du bacille tuberculeux à partir des produits biologiques par l'emploi des sels d'ammonium quaternaire ", Ann. Inst. Pasteur Lille, 1956, 8 : 89-94.

4. LANGEROVA (M.) et TACQUET (A.), « Comparaison de différentes méthodes d'homogénéisation et de purification des produits pathologiques en fonction de milieux de culture solides et liquides », Bull. O.M.S., 1968, 39: 663-80. 
5. SARRAT (H.), «Emploi du bromure de cétylpyridinium comme milieu de transport des produits pathologiques en vue de l'isolement de mycobactéries », Méd. Afr. noire, 1970, 17 (4): 333-37

6. TACQUET (A.) et TISON (F.), « Résultats de l'isolement de bacilles tuberculeux dans 55.000 crachats par le Teepol 410 basique. Influence du milieu de culture », Ann. Inst. Pasteur, 1961, 100 (2) : 153-58

7. TACQUET (A.) et TISON (F.) * Nouvelle technique d'isolement des mycobactéries par le lauryl sulfate de sodium $»$, Ann. Inst. Pasteur, 1961, 100: 676-80.

8. TACQUET (A.), TISON (F.) et MARTIN (G.), « Etudes de divers procédés d'épuration des produits pathologiques et milieux de culture pour l'isolement des mycobactéries ", Ann. Inst. Pasteur Lille, 1962, 13 : 57-76.

9. TACQUET (A.), TISON (F.) et POLSPOEL (B.),
* L'utılisation des détergents pour l'isolement des mycobactéries à partir de produits patholog1. ques », Ann. Inst. Pasteur Lille, 1966, 16: 21-30.

10. TACQUET (A.) et collab., *Technique de recherche des mycobactéries dans le lait et les produits laitiers », Ann. Inst. Pastew Lille, 1966, 17 : 161-72.

11. TISON (F.), «Nouveau procédé d'isolement du bacille tuberculeux dans les crachats. Usage du Teepol dilué », Ann. Inst. Pasteur, 1954, 87: 735-36.

12. TISON (F.) et collab., «Technique et résultats de la recherche des mycobactérnes dans les viandes », Ann. Inst. Pasteur Lille, 1966, 17: 155-60.

13. TISON (F.) et collab., "La décontamination des produits pathologiques en vue de la culture des mycobactéries. Etude comparative des méthodes actuelles », Ann. Inst. Pasteur, 1967, 112 (3): 372-78. 